\title{
BMJ Open Use of a computerised decision aid (DA) to inform the decision process on adjuvant chemotherapy in patients with stage II colorectal cancer: development and preliminary evaluation
}

\author{
A Miles, ${ }^{1}$ I Chronakis, ${ }^{2}$ J Fox, ${ }^{2,3}$ A Mayer ${ }^{4}$
}

To cite: Miles A, Chronakis I, Fox J, et al. Use of a computerised decision aid (DA) to inform the decision process on adjuvant chemotherapy in patients with stage II colorectal cancer: development and preliminary evaluation. BMJ Open 2017;7:e012935. doi:10.1136/bmjopen-2016012935

- Prepublication history and additional material is available. To view please visit the journal (http://dx.doi.org/ 10.1136/bmjopen-2016012935).

Received 3 June 2016 Revised 26 September 2016 Accepted 8 November 2016

CrossMark

For numbered affiliations see end of article.

Correspondence to

Dr A Miles;

ae.miles@bbk.ac.uk

\section{ABSTRACT}

Objectives: To develop a computerised decision aid (DA) to inform the decision process on adjuvant chemotherapy in patients with stage II colorectal cancer, and examine perceived usefulness, acceptability and areas for improvement of the DA.

Design: Mixed methods.

Setting: Single outpatient oncology department in central London.

Participants: Consecutive recruitment of 13 patients with stage II colorectal cancer, 12 of whom completed the study. Inclusion criteria were: age >18 years; complete resection for stage II adenocarcinoma of the colon or rectum; patients within 14-56 days after surgery; no contraindication to adjuvant chemotherapy; able to give written informed consent. Exclusion criterion: previous chemotherapy.

Primary outcomes: Patient perceived usefulness (assessed by the PrepDM questionnaire) and acceptability of the DA.

Results: PrepDM scores, measuring the perceived usefulness of the DA in preparing the patient to communicate with their doctor and make a health decision, were above those reported in other patient groups. Patient acceptability scores were also high; however, interviews showed that there was evidence of a lack of understanding of key information among some patients, in particular their baseline risk of recurrence, the net benefit of combination chemotherapy and the rationale for having chemotherapy when cancer had apparently gone.

Conclusions: Patients found the DA acceptable and useful in supporting their decision about whether or not to have adjuvant chemotherapy. Suggested improvements for the DA include: sequential presentation of treatment options (eg, no treatment vs 1 drug, 1 drug vs 2 drugs) to enhance patient understanding of the difference between combination and single therapy, diagrams to help patients understand the rationale for chemotherapy to prevent a recurrence and inbuilt checks on patient understanding of baseline risk of recurrence and net benefit of chemotherapy.

\section{Strengths and limitations of this study}

- This study evaluated patient acceptability, perceived usefulness and understanding of a decision aid (DA) using mixed-methods at the point patients made their decision about whether or not to have adjuvant chemotherapy.

- We recruited men and women with a range of different ages and levels of education, including people with no educational qualifications, enabling us to capture a range of different responses to the DA.

- People educated to degree level or above were over-represented in the study, and this may have led to an overestimate of perceived usefulness and acceptability of the DA.

\section{INTRODUCTION}

Ethical considerations and recent policy changes have put patients at the centre of health decisions, aiming to make shared-decision-making 'the norm'. ${ }^{2}$ One key issue for people who have undergone surgical resection of colorectal cancer is the decision about whether or not to have adjuvant chemotherapy, which is typically either 5-FU or capecitabine, given alone or in combination with oxaliplatin. ${ }^{3}{ }^{4}$ While surgery is highly effective for localised disease, up to $85 \%$ of patients with lymph node involvement (stage III) relapse within 5 years. Since chemotherapy can prevent recurrence in up to $25 \%$ of patients with stage III disease, ${ }^{5}$ it is offered routinely. For patients without lymph node involvement (stage II), the risk of relapse is lower $(20-40 \%)$ and a smaller number of patients $(3-7 \%)$ are expected to benefit. ${ }^{67}$ Since there is a risk of side effects following chemotherapy, some of which can be life-threatening or permanent and the balance of harms and benefits is marginal in 
patients with stage II, the use of adjuvant chemotherapy in this patient group has remained controversial. ${ }^{8}$

Patients with colorectal cancer have expressed a desire for more information, notably about their cancer, prognosis and treatment options, ${ }^{9} 10$ and effective communication of diagnostic and prognostic information has been shown to enhance patient well-being and patients' perceptions of the quality of doctor-patient interactions. ${ }^{11}$ However, patients can hold more positive views on adjuvant therapy than physicians ${ }^{12}$ and may want chemotherapy even when there is little evidence that it will help prevent a recurrence; ${ }^{13}{ }^{14}$ for example, women with breast cancer with a low risk of recurrence are more likely to decline chemotherapy if they are aware of the (small) impact, ${ }^{15}$ underlining the need for effective communication. People have problems understanding risk information, ${ }^{16}$ and while there is an emphasis on patients making decisions that are consistent with their values, evidence points to the instability of preferences, with some researchers arguing that true preferences may not always exist, but are constructed 'on the spot' using the information available at the time of decision-making. ${ }^{17}$

\section{Decision aids}

To facilitate informed decision-making, a wide range of decision aids (DAs) have been developed. DAs are used alongside patient-physician interactions and contain detailed information about clinical options and outcomes, are explicit about the choices facing the patient and encourage patients to express their preference. DAs typically result in greater knowledge, a higher proportion of people with accurate risk perceptions, lower decisional conflict (eg, feeling better informed and clearer about personal values) and more active roles in decisionmaking compared with usual care. ${ }^{18}$ Although DAs have been developed to aid treatment decisions for a number of different cancers, ${ }^{19-22}$ little research has been done in the context of colorectal cancer. One exception is a booklet to help patients make decisions about adjuvant chemotherapy or radiotherapy, although this was only evaluated among patients who had opted to have adjuvant therapy, and was developed to be used by the patient at home rather than as part of a clinical consultation. ${ }^{10} 23$

The aim of the present study was to develop a DA for chemotherapy for stage II that included the information identified as important to patients in previous research $^{910}$ and examine its usefulness and acceptability to patients making a decision about whether or not to have adjuvant chemotherapy, as well as to identify any areas where patient understanding of key information could be improved.

\section{METHODS}

Development of the DA

PROforma decision support technology (a formal language for automating clinical processes and protocols) and the Tallis tool set (used to design the decision support, check the syntactic integrity of the programme and run the application in a test environment) were used to develop the DA; ${ }^{24}{ }^{25} \mathrm{http}: / /$ openclinical.net/ index.php?id=390. A variety of decision support applications based on PROforma technology have demonstrated a significant positive impact on a number of outcome measures such as reducing prescription errors and adherence to evidence-based guidelines. ${ }^{26-28}$

The DA captured patient demographic and clinical information (eg, histology type, evidence of extramural vascular invasion), as well as patient fitness to undergo chemotherapy (assessed via the presence of comorbidity and ECOG performance status ${ }^{29}$ ranging from fit and well (0) to bed bound (4)).

Risk of recurrence with and without chemotherapy was mathematically derived based on: (1) the baseline risk of recurrence without chemotherapy, which was calculated for each patient using information from the published SEER database (http://seer.cancer.gov/data/) using the adjuvant!online risk calculator (http://www. adjuvantonline.com); $;^{30}$ and (2) the effectiveness of single and combination chemotherapy, taken from published randomised controlled trials and meta-analysis. ${ }^{6} 7$ This calculator matches baseline risk of recurrence data to the individual patient's clinicopathological characteristics ( $\mathrm{T}$ stage, number of lymph nodes examined, histology type and histopathological grading). The age-specific natural mortality was derived from published national mortality data.

In line with the presentation of information in Adjuvant!online, and consistent with recommendations, we used a simple percent format, and absolute risk when conveying information about the net benefit of chemotherapy along with a bar chart showing the likelihood of different outcomes, thereby using visual and numeric information to convey risk of recurrence information, keeping the denominator consistent and stating the reference class and time frame. ${ }^{31} 32$

Patients were first shown one graph detailing their baseline risk of recurrence in the absence of chemotherapy (see figure 1) and graphs showing the net benefit of single and combination chemotherapy on the final page of the DA (see figure 2). Since the risk prediction of Adjuvant!online does not include clinical presentation (obstruction or perforation), or the risk factors vascular, lymphovascular and perineural invasion, these were included under headings for 'indicators for good prognosis' (eg, tumour stage T3) and 'indicators for bad prognosis' (eg, extramural vascular invasion), presented on the final page of the DA (see figure 2).

Information about the method of administration for the different drugs, the need for the insertion of a central line, the number of times the patient would need to come to hospital and the duration of treatment was given. The list of side effects were put into three different sections: common ( $>30 \%$ of patients affected), less common but serious (potentially life-threatening) 


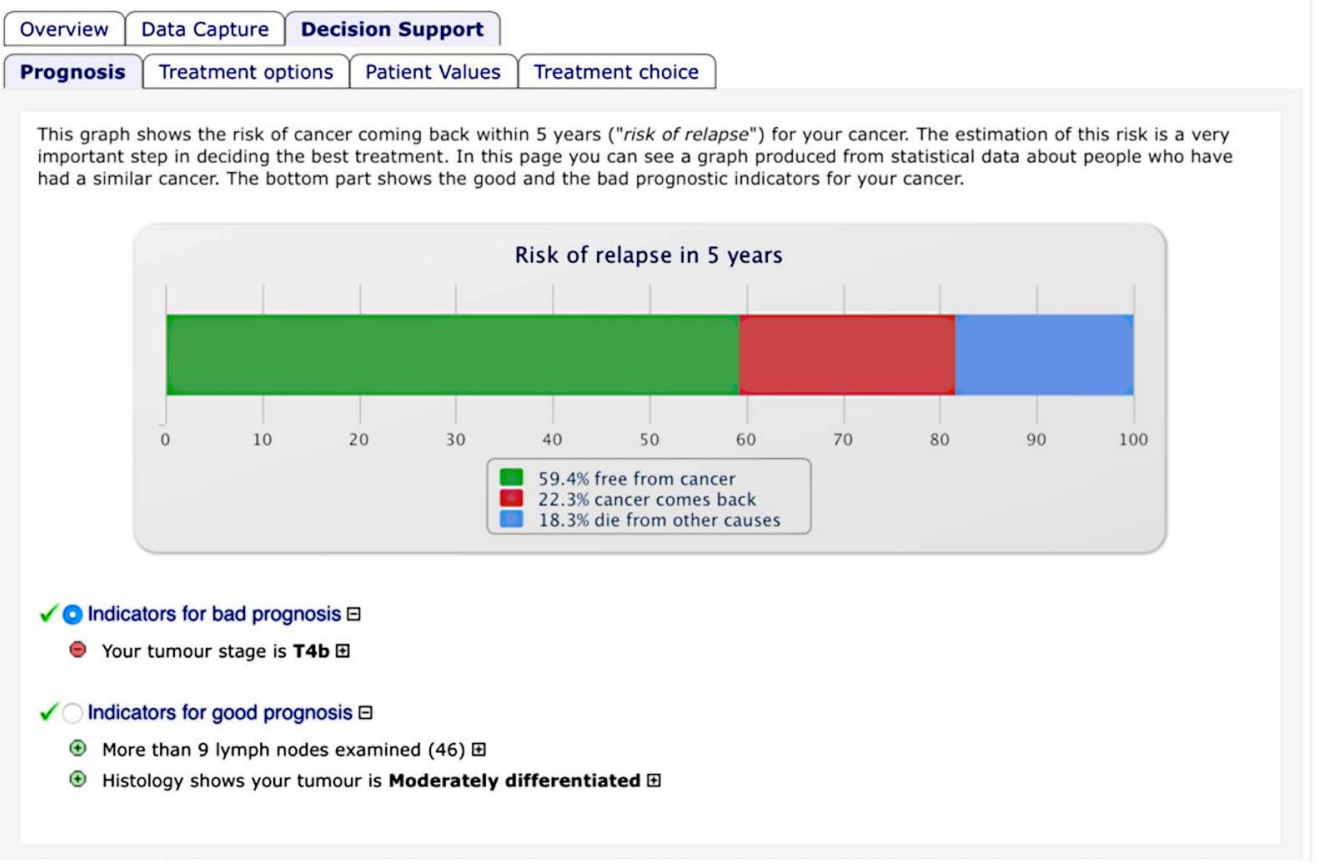

Figure 1 Graph showing baseline risk of recurrence.

and potentially permanent side effects. It was made clear that the first two sections related to all chemotherapy options, but that the long-term side effects only related to the two-drug chemotherapy option (oxaliplatin carries a high risk of long-term numbness of the hands and feet). ${ }^{4}$

The values clarification exercise was adapted from one developed for use in colorectal cancer screening among elderly people ${ }^{33}$ to capture the main issues involved in the decision, including features identified as important to patients with colorectal cancer ${ }^{9}$ (see online supplementary table S1). Patients were asked to complete the questionnaire on the computer, selecting 1 of the 2 options presented for all 10 questions. Patients were able to review and change their responses to the questions at any time during or after the consultation.

The final page of the DA combined graphical presentation of risk of recurrence information for the three treatment options alongside arguments laying out the pros and cons for each option, and the results of the values clarification exercise (see figure 2 ).

\section{Design}

A mixed methods design was employed.

\section{Participants}

Potential patients were identified at the colorectal multidisciplinary team meeting at the Royal Free NHS Trust. Inclusion criteria were: male or female patients aged $>18$ years; patients who had undergone complete resection for stage II adenocarcinoma of the colon or rectum; patients within 14-56 days after surgery; no contraindication to adjuvant chemotherapy; able to give written informed consent. The exclusion criterion was previous chemotherapy. Target $\mathrm{N}$ was 12 in line with similar research on DAs. ${ }^{34}$ Patients were recruited consecutively.

\section{Procedures}

After informed consent, patients were randomised to receive the DA at either the first or second consultation. All patients were given written information about the side effects of chemotherapy, as per current practice. When seen back in clinic, after 1-2 weeks, patients were given the opportunity to ask questions, at which point the patient's treatment decision was recorded. Patients were invited to complete the values clarification exercise on the computer, but received help from relatives or the clinician if requested.

All consultations took place in the Outpatients Oncology Department at the Royal Free London NHS Trust. Patients were given a questionnaire assessing responses to the DA after the consultation at which the DA had been used.

\section{Questionnaire}

Information about age, gender, educational level, ethnicity and employment status was collected. Perceived usefulness of the DA in preparing the patient to communicate with their doctor and make a health decision was assessed with the PrepDM questionnaire, a 10-item scale designed to measure the usefulness of DAs, developed across a number of different patient groups, including patients with cancer, with good internal reliability and validity. ${ }^{35}$ Cronbach's $\alpha$ in this study was 0.935 . Example items include: 'Did the decision aid...Help you 


\section{Information}

Here you can see how the available options compare with each other. In addition you will see how each option matches you personal preference as it has been entered in the previous questionnaire.

\section{Icon explanation}

(†) This is a reason supporting an option

- This is a reason opposing an option

8 This option is in line with your preferences

8 This option is against your preferences

\section{- No addtional chemotherapy \\ 5 year risk of relapse with no chemotherapy \\ 0

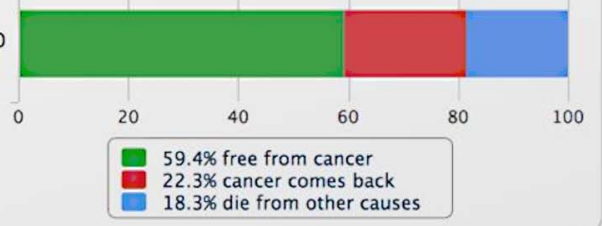 \\ One chemotherapy drug}

(†) Quality of life is not affected. You can expect to live free of any commitments and interventions due to chemotherapy.

(4) Patients older than 70 years have been reported to benefit less from chemotherapy $⿴ 囗 十$

8 The results of your value clarification exercise are 10 in favour and 0 against No addtional chemotherapy. $\boxplus$

The drug in this case is either 5FU or Capecitabine. Both of them are equally effective but some patients are suited better for the former and others for the latter. Your clinician will be able to give you advice regarding which option is better for you so that you can balance the side effects with the benefits.

Reduction of 5 year risk with one chemotherapy drug

0

0

\begin{tabular}{|l|l|}
\hline $20 \quad 40 \quad 100$ \\
$59.4 \%$ free from cancer \\
$3.6 \%$ free from cancer due to chemotherapy \\
$18.2 \%$ cancer comes back \\
$18.8 \%$ die from other causes
\end{tabular}

(†) The reduction of risk of relapse by one chemotherapy drug is $3.6 \%$

$(\oplus$ You are reasonably fit, chemotherapy can potentially tolerated well. 田

(4) There are 1 bad prognostic indicators present. $⿴$

- Chemotherapy will require a commitment for a considerable amount of time (6 months) involving reqular hospital visits and taking medication with potential side effects. $\rightarrow$

8 The results of your value clarification exercise are 0 in favour and 9 against One chemotherapy drug. 1 .

\section{Two chemotherapy drugs}

This option consists of the combination of 5FU or Capecitabine with Oxaliplatin. As in the case of the one chemotherapy drug, your consultant will advice you whether $5 F U$ or Capecitabine is more suitable for you.

Reduction of 5 year risk with two chemotherapy drugs

$$
0
$$

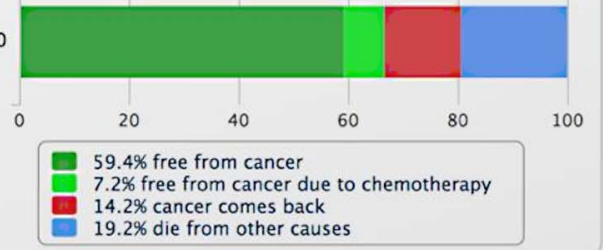

(†) The reduction of risk of relapse by the two chemotherapy drugs is $\mathbf{7 . 2} \%$

(†) You are reasonably fit, chemotherapy can potentially tolerated well. $⿴ 囗 十$

Patients older than 70 years have been reported to benefit less from chemotherapy $⿴ 囗 十$

$\oplus$ There are 1 bad prognostic indicators present. $⿴$

- Chemotherapy will require a commitment for a considerable amount of time, usually 6 months, involving regular hospital visits and taking medication with potential side effects. $\rightarrow$

8 The results of your value clarification exercise are 1 in favour and 10 against Two chemotherapy drugs. $\boxplus$

Figure 2 Final page of decision aid showing risk of recurrence and net benefit of treatment options alongside pros and cons of different treatment options.

think about the pros and cons of each option? ... Help you identify questions you want to ask your doctor?' Response options are on a five-point Likert scale with higher scores indicating higher agreement. Acceptability of the DA was assessed using seven items; three were from Bennett et $a l,{ }^{35}$ while the remaining items were developed for this study (see table 1 for questions and response options). Data were analysed using SPSS V.22.

\section{Interview}

Face-to-face interviews were used to gain an in-depth understanding of patients' responses to the DA, and their understanding of key information. These were conducted face to face at the hospital following the second consultation, between March 2012 and July 2014, by AMi, who has experience of conducting interviews with patients with colorectal cancer, ${ }^{36}$ and was not involved in the patients' care. The interviews lasted an average of $33 \mathrm{~min}$ (range: 18-54) and were recorded and transcribed verbatim by a transcription company, and reviewed for accuracy by AMi using the original recordings. The initial Topic Guide is provided as online supplementary data, but during the interviews, it became clear that patients were confused about what 
Table 1 Perceived usefulness and acceptability of the decision aid

\begin{tabular}{|c|c|}
\hline & Number \\
\hline $\begin{array}{l}\text { Usefulness of information in preparing patient } \\
\text { for decision (PrepDM) }(1-5) \text { mean (SD) }\end{array}$ & $4.28(0.90)$ \\
\hline \multicolumn{2}{|l|}{ Acceptability of $D A^{35}$} \\
\hline \multicolumn{2}{|l|}{ Helpful } \\
\hline Very & 10 \\
\hline Somewhat & 1 \\
\hline A little & 1 \\
\hline Not helpful & 0 \\
\hline \multicolumn{2}{|l|}{ Recommend to others } \\
\hline Definitely yes & 6 \\
\hline Probably yes & 5 \\
\hline Probably not & 1 \\
\hline Definitely not & 0 \\
\hline \multicolumn{2}{|l|}{ Information clear } \\
\hline Everything clear & 8 \\
\hline Mostly clear & 4 \\
\hline Some clear & 0 \\
\hline Mostly unclear & 0 \\
\hline \multicolumn{2}{|l|}{ Did the DA add anything to the consultation } \\
\hline An extreme amount & 1 \\
\hline Very much & 7 \\
\hline A moderate amount & 4 \\
\hline A little bit & 0 \\
\hline Nothing & 0 \\
\hline \multicolumn{2}{|l|}{ Graphical representation in the DA helpful } \\
\hline Extremely & 4 \\
\hline Very & 6 \\
\hline Moderately & 2 \\
\hline A little bit & 0 \\
\hline Not at all & 0 \\
\hline \multicolumn{2}{|l|}{ Understood risk of recurrence info. in DA } \\
\hline Yes & 10 \\
\hline No & 0 \\
\hline Not sure & 1 \\
\hline \multicolumn{2}{|l|}{ Helpfulness of risk of recurrence info. in DA } \\
\hline Extremely & 3 \\
\hline Very & 6 \\
\hline Moderately & 1 \\
\hline A little bit & 1 \\
\hline Not at all & 0 \\
\hline
\end{tabular}

risk of recurrence meant and how chemotherapy could act on the body to prevent this. Subsequent patients were therefore asked about these issues in more detail. Data were analysed using thematic analysis, ${ }^{37}$ facilitated by the software package NVIVO V.10. AMi developed the codes by examining all instances in the data set that related to the DA, risk of recurrence information and the rationale for having chemotherapy. Themes were derived and developed via an iterative process, with constant comparisons of the data that identified similarities and differences within and across individual interviews. The codes and supporting quotes were reviewed for appropriateness and accuracy by AMa. Patients were not asked to verify the thematic analysis as we did not wish to increase participation burden.

\section{RESULTS}

Fifteen patients were approached by clinic staff, 13 were recruited, with 12 completing the study. The age of participants ranged from 33 to 82 , with a median age of 67 . The ratio of men to women was 1:2. Over half $(n=7)$ were educated to degree level or equivalent and the majority were of white ethnicity (British or Irish) $(\mathrm{n}=9)$. Approximately half were employed $(n=6)$, one was unemployed and the remainder were retired. One patient reported having had chemotherapy prior to surgery (s6). This person was included since it was not felt to impact on the reported outcomes. Eleven of the 12 patients declined chemotherapy (all except s8).

Patients' perceptions of the usefulness of the DA in helping them communicate with their doctor and make a decision were good, with scores (mean 4.28, SD 0.9) comparing favourably with those reported in other clinical populations, where scores ranged from 3.3 to $3.9 .^{35}$ Patients also reported finding the risk of recurrence information in the DA and graphical representation of information in the DA helpful, with all except one reporting that they were happy they understood the meaning of the information in the DA about risk of recurrence (see table 1).

\section{Graphical representation of information on risk}

A number of patients felt the graphs increased the clarity of the risk of recurrence information, "I liked the graphs, I suppose they're accurate really... I worked with statistics enough to know that you can kind of hide the numbers.... it made me consider chemotherapy more than I possibly would if I was just given words" (s10). Another participant described how the graphical presentation looked more scientific and hence more credible than being told the same information verbally: "Really it's no different to what the surgeon had already told us yesterday... it's a very much more scientific, you know, sort of presentation to it..." (s4).

For some patients, the graphs provided information they would otherwise have overlooked: for one patient, it was the additional benefit conferred by two, compared with one, chemotherapy drug "I didn't realise that the one was quite like a significant difference there, between the two (chemotherapy drugs)" (s3); for another patient, it was the risk of death from other causes: "When we're there we only talk about coming back and not coming back. But we do know that death is something that can come anytime... So I just put them in the two categories but on the graph I saw the three there" (s9).

Some patients found it helpful to see the portion of the graph that illustrated the net benefit of chemotherapy, whether they wanted chemotherapy or not, and used it to help them cope with their decision: "that made me feel quite positive... even though there was only $5 \% \ldots$ I'm going into the chemotherapy, now, thinking about that line" (s8), "the graph...the bit that she 
was talking about (net benefit of chemotherapy) is just tiny is quite good" (s3).

Some patients liked the positive information about their general prognosis- "I was quite pleased to see that. That was nice. There was lots of green (baseline risk of recurrence)" (s8). One person liked it because they described themselves as 'a visual person' (s3).

However, not everyone was able to articulate whether or why they had found the graphical information helpful, particularly if they had taken a younger relative into the consultation with them and felt confident their relative understood what was being said. "I didn't really understand it very much...I'm a bit of a dinosaur... I know my niece would have understood it all" (s11), "I just can't say...My son was with me now and he was very informative afterwards, he was, talked about it a lot...I think he found it very helpful" (s12).

\section{Values clarification}

The belief that they could not predict whether they would benefit from chemotherapy, the small risk of cancer coming back and the long-term side effects of chemotherapy were the main barriers to chemotherapy (see online supplementary table S2). Of least concern were the short-term side effects, insertion of a central line and repeat attendance at hospital, although around half were still concerned about these things.

Reactions to the values clarification exercise were more mixed than to the graphical display. Some patients found it helpful, while others reported conflicting feelings about the information. Positive reactions included the feeling that it was an accurate reflection of their (often mixed) views about chemotherapy, was clearer, more explicit, more rational and less emotional, and empowering for the patient: "it's good in terms of sometimes you struggle to articulate why you make one decision versus another" (s10) "getting me to kind of click on things, and say yes... puts the onus on me to make a decision and own my decision and feel empowered to make a decision independent of what the doctor thinks..." (s8). "Rather than having an emotional response, there's an actual figure there to say, 'This is what you decided.' ...it's good to be logical" (s3).

Others commented on the fact that not all the items were equally important and that numbers for and against chemotherapy were a bit simplistic, or having to select a choice yes/no without saying why did not tell the whole story.

One patient reported feelings of guilt when answering the values clarification exercise, because although they viewed their health as important they were opting to do something that did not necessarily maximise their chances of maintaining their health: "my health comes first above everything. And so a lot of questions there made me feel when I opted for a different answer to that, to really putting that first, I felt very guilty about, which was quite interesting. And I did pause and stress about that a little bit... But just because I answered the other way doesn't mean I still don't have my health, you know, at the forefront of my thoughts... However, there are other issues at stake here" (s4).

Another felt it highlighted the importance of life over everything else: "I'm worrying about hair loss and this and that.... And what about that? Is that more important than life itself? Well, no, it isn't... But that tool...did think, you know, all of that is trivial in comparison with life itself. It's very precious" (s7).

However, one woman felt she only needed the risk of recurrence statistics to make a decision, and did not need the values clarification exercise: "It didn't seem to be difficult to answer really.... the biggest thing that sticks out in my mind is the statistics and the fact that taking the chemo again made really very little difference, that my mind was pretty well made up straight away" (s6). Others forgot whether or not they had performed the exercise: "In all honesty, I'd forgotten that, you know. I don't remember I did or not do" (s11).

\section{Patient understanding of risk of recurrence statistics}

The majority of patients understood that the aim of chemotherapy was to reduce the chances of cancer coming back, although people were more likely to mention the figure for net benefit of one chemotherapy drug (2-3\%) rather than two (5\%): "if I had chemotherapy, my chances of living more than five years would be about one or two percent increased, compared with not doing anything" (s1). "The chance of the cancer coming back was low-without the chemo, was low, anyway... Yes, the chemo might improve it by a couple of percent" (s4).

Two patients (both in their 30s) reported higher net benefit of chemotherapy figures (of $5 \%$ ) and a clearer understanding of the difference between one and two chemotherapy drugs. "I think off the top of my head there was $86 \%$ chance of it not recurring within five years if I stopped it now... then for every additional drug chemo that I had was approximately a $2.5 \%$ additional chance of survival" (s10). One patient confused net benefit (additional 2-5\% avoid recurrence) with overall recurrence rates (20-40\%). "(Oncologist's name) said to me, 'There's just 2\% chance.' When I'd said, 'No, I do not want chemo,'.... I took for granted that sort of the chances of it coming back is extremely low" (s11).

\section{Patient understanding of how and why cancer recurs and} how chemotherapy may act to reduce risk of recurrence

There was confusion over whether the figures about recurrence referred to another primary cancer or progression of the current episode, or both. "Was it a recurrence of the same cancer or was it from somewhere else?" (s6). While some patients understood that any recurrence would potentially be more aggressive and harder to treat than their colorectal cancer had been, others assumed the recurrence would be in the gut: "When you say it's a recurrence, you presume it will be 
expected to be from the same site where the primary is... somewhere in the gut..." (s6). "Actually, in the beginning I was thinking that they were talking about that, the cancer will affect my bowel again. But from what she's (the oncologist) saying...it's not only the bowel, but you can get other type of cancer" (s9). "Obviously if it comes back it's not going to be as straightforward... It wouldn't be as simple as just having an operation and removing it, cos it might go to another part of the body" (s7).

An additional area of confusion was whether they were currently cancer free or not, and the rationale for giving chemotherapy when no cancer was apparently present or if you were a person 'prone' to cancer. "People say that you're 'cured' now because the cancer's been removed surgically. But then you've got this risk of recurrence. I found that quite difficult to wrestle in my head because it doesn't feel like I'm cured. It feels like I've got cancer and I've got to see what happens over the next five years..." (s8). "But that has been my problem. Even taking the chemotherapy, if you're talking about, well if cancer, prone to cancer. If cancer will come and chemotherapy wouldn't.... if it were there, I agree it would affect it, but if you will come back I don't think chemotherapy can stop it. That's my understanding; maybe I'm wrong" (s9). But some patients did not think their cancer would come back, and this belief seemed to be the basis of their decision to refuse chemotherapy: "I'm sure that if I thought there was a possibility of it coming back, for me, I would.... I would go for treatment. But I'm just hoping that it doesn't happen" (s12).

\section{DISCUSSION}

We developed a DA for patients with stage II colorectal cancer to facilitate an informed decision process for adjuvant chemotherapy. Patient perceptions of the usefulness of the DA were above scores reported in other patient groups, ${ }^{35}$ but there was evidence of a lack of understanding of key information among some patients, in particular: their baseline risk of recurrence, the net benefit of combination chemotherapy and the rationale for having chemotherapy when cancer had apparently gone.

The method of communicating risk of recurrence information based on Adjuvant!online improves patient understanding of the likelihood of disease-free survival. $^{15}$ Numeric information about risks enhances patient understanding. ${ }^{38}$ While visual displays can be very helpful, not everyone can extract the relevant information from them. ${ }^{31}$ Consistent with previous research, ${ }^{15}$ we found that despite presenting numeric and visual risk information, there was evidence of a lack of understanding of risk information among some patients.

Research on members of the general population has shown that understanding of the graphical risk information given in Adjuvant! online is improved if people are presented with fewer options at any one time, ${ }^{39}$ and that sequential presentation of treatment options leads to improved understanding of key information about risk of recurrence and net benefit of treatment. ${ }^{40}$ In this study, patients were presented with all three options at once, and patient understanding of combination chemotherapy might have been enhanced by presenting just two options at a time (eg, no treatment vs one drug, no treatment vs two drugs). In addition, any benefit of single agent chemotherapy has only been observed among patients with bad prognostic factors, ${ }^{4}$ which may be limited to T4. ${ }^{41}{ }^{42}$ Significant efforts are being made to develop better prognostic indicators including the search for gene signature sets that predict response to chemotherapy in this patient group. ${ }^{43}$ This suggests that, in future, even more complex information may need to be presented to patients.

The values clarification exercise required patients to select arguments for and against chemotherapy. Although the pros and cons method is the most commonly used method for values clarification purposes, a variety of different methods are available, such as ranking, and social matching (how another person values the characteristics of different options and how similar that person is to you), but owing to a lack of research, there are no explicit recommendations about best practice, and no firm evidence about how and whether values clarification exercises actually inform and help the decision-making process. ${ }^{45}$ Patient feedback showed that some patients found the exercise useful, and for one clarified the bottom line of the decision: prolonging life versus experiencing side effects. However, for others, the process was less helpful, either because understanding of the information contained in the DA had effectively been delegated to a younger member of the family (niece/son) or because the risk of recurrence information was all they felt they needed to make a decision. There is a lack of research about which patients may benefit from values clarification exercises, and the best methods to engage people with different cognitive ability or lower literacy levels and these issues remain to be explored. ${ }^{45}$ The values clarification exercise used in this study had a higher than recommended readability level, and a simpler method of eliciting patient preferences will be needed for patients with lower levels of literacy.

A further suggested modification of the DA is the addition of information that helps people understand the rationale for being given chemotherapy when cancer has apparently gone. The information booklet developed by Jefford $e t a l^{10}$ included diagrams showing how colorectal cancer can spread around the body, and similar information should be included in a revised DA.

A key limitation of this study is that patients educated to degree-level or above were over-represented, which may have led to an overestimate of perceived usefulness 
and acceptability of the DA as measured by the PrepDM and other questionnaire items.

The American Cancer Society estimated that a third of people diagnosed with colon cancer in 2013 will be diagnosed at stage $\mathrm{II}^{8}$, resulting in a substantial number of patients potentially faced with making a decision about adjuvant chemotherapy. It is clear that challenges remain in communicating risk of relapse information to patients and the need to check patient understanding of key information should be integrated in future DAs, for example, with the insertion of quizzes and representation of key information that patients appear to have misunderstood. In addition, much more research is needed into the best way to facilitate patients in making judgements about what is most important to them through a greater understanding of values clarification methods in assisting complex decisions.

\section{Author affiliations}

${ }^{1}$ Birkbeck, University of London, London, UK

${ }^{2}$ University College London, London, UK

${ }^{3}$ Oxford University, Oxford, UK

${ }^{4}$ Royal Free London NHS Trust, London, UK

Twitter Follow loannis Chronakis @chronakis

Contributors AMa (guarantor) had the original idea for the study. AMa, IC and $\mathrm{AMi}$ designed the trial variables and formed the investigator group which obtained the funding. AMa, IC, JF and AMi developed the decision aid. AMa and AMi were responsible for overseeing study implementation and data collection. They also carried out the analysis, and drafted the manuscript, which was revised by all authors.

Funding This research was supported by the Royal Free Charity.

Disclaimer All researchers were independent of the funders. The study sponsor and funder played no role in study design; the collection, analysis and interpretation of data; the writing of the report; and the decision to submit the article for publication.

Competing interests Over the duration of the project, IC was funded by a grant from the Royal Free Charity and employed by UCL. The Royal Free Charity funded the development of the EPAD software. IC is now employed by Deontics. He is a founder and a shareholder of the company. Deontics is a commercial provider of clinical decision support software. This company was set up after the study reported in the paper was completed. ePAD (the DA tool) was developed using software now owned by Deontics. Over the duration of the project, JF was employed by UCL and Oxford Universities. The Royal Free Charity funded the development of the ePAD software. JF is now employed part-time by Deontics. He is a founder and a shareholder of the company.

Ethics approval Ethical approval was given by the London Multi-Centre Research Ethics Committee (REC 11/LO/0888).

Provenance and peer review Not commissioned; externally peer reviewed.

Data sharing statement No additional data are available.

Open Access This is an Open Access article distributed in accordance with the Creative Commons Attribution Non Commercial (CC BY-NC 4.0) license, which permits others to distribute, remix, adapt, build upon this work noncommercially, and license their derivative works on different terms, provided the original work is properly cited and the use is non-commercial. See: http:// creativecommons.org/licenses/by-nc/4.0/

\section{REFERENCES}

1. TSO. UK Department of Health. Equity and excellence: liberating the NHS. 2010.
2. General Medical Council. Consent: patients and doctors making decisions together. 2016.

3. Twelves C, Wong A, Nowacki MP, et al. Capecitabine as adjuvant treatment for stage III colon cancer. N Engl J Med 2005;352:2696-704.

4. André $\mathrm{T}$, Boni $\mathrm{C}$, Navarro $\mathrm{M}$, et al. Improved overall survival with oxaliplatin, fluorouracil, and leucovorin as adjuvant treatment in stage II or III colon cancer in the MOSAIC trial. J Clin Oncol 2009;27:3109-16.

5. de Gramont A, Larsen AK, Tournigand C, et al. Update on targeted agents for adjuvant treatment of colon cancer in 2006. Gastrointest Cancer Res 2007;1(Suppl 2):S47-9.

6. Gray R, Barnwell J, McConkey C, et al. Adjuvant chemotherapy versus observation in patients with colorectal cancer: a randomised study. Lancet 2007;370:2020-9.

7. Kuebler JP, Wieand HS, O'Connell MJ, et al. Oxaliplatin combined with weekly bolus fluorouracil and leucovorin as surgical adjuvant chemotherapy for stage II and III colon cancer: results from NSABP C-07. J Clin Oncol 2007;25:2198-204.

8. Fang $\mathrm{SH}$, Efron JE, Berho ME, et al. Dilemma of stage II colon cancer and decision making for adjuvant chemotherapy. J Am Coll Surg 2014;219:1056-69.

9. Sanoff HK, Goldberg RM, Pignone MP. Assessing the quality of initial consultations regarding adjuvant colon cancer therapy. Clin Colorectal Cancer 2010;9:113-18.

10. Jefford M, Gibbs A, Reading D. Development and evaluation of an information booklet/decision-making guide for patients with colorectal cancer considering therapy in addition to surgery. Eur J Cancer Care (Engl) 2005;14:16-27.

11. Repetto L, Piselli P, Raffaele M, et al. Communicating cancer diagnosis and prognosis: when the target is the elderly patient-a GIOGer study. Eur J Cancer 2009;45:374-83.

12. Harrison JD, Solomon MJ, Young JM, et al. Patient and physician preferences for surgical and adjuvant treatment options for rectal cancer. Arch Surg 2008;143:389-94.

13. Ravdin PM, Siminoff IA, Harvey JA. Survey of breast cancer patients concerning their knowledge and expectations of adjuvant therapy. $J$ Clin Oncol 1998;16:515-21.

14. Duric VM, Stockler MR, Heritier S, et al. Patients' preferences for adjuvant chemotherapy in early breast cancer: what makes AC and CMF worthwhile now? Ann Oncol 2005;16:1786-94.

15. Belkora JK, Hutton DW, Moore DH, et al. Does use of the adjuvant! model influence use of adjuvant therapy through better risk communication? J Natl Compr Canc Netw 2011;9:707-12.

16. Gigerenzer G, Edwards A. Simple tools for understanding risks: from innumeracy to insight. BMJ 2003;327:741-4.

17. Reyna VF, Nelson WL, Han PK, et al. Decision making and cancer. Am Psychol 2015;70:105-18.

18. Stacey D, Legare F, Col NF, et al. Decision aids for people facing health treatment or screening decisions. Cochrane Database Syst Rev 2014;(1):CD001431.

19. Adsul $P$, Wray R, Spradling $K$, et al. Systematic review of decision aids for newly diagnosed patients with prostate cancer making treatment decisions. J Urol 2015;194:1247-52.

20. Jones RA, Steeves R, Ropka ME, et al. Capturing treatment decision making among patients with solid tumors and their caregivers. Oncol Nurs Forum 2013;40:E24-31.

21. Sawka AM, Straus S, Gafni A, et al. A usability study of a computerized decision aid to help patients with, early stage papillary thyroid carcinoma in, decision-making on adjuvant radioactive iodine treatment. Patient Educ Couns 2011;84:e24-7.

22. Sawka AM, Straus S, Rotstein L, et al. Randomized controlled trial of a computerized decision aid on adjuvant radioactive iodine treatment for patients with early-stage papillary thyroid cancer. J Clin Oncol 2012;30:2906-11.

23. Juraskova I, Butow P, Fisher A, et al. Development and piloting of a decision aid for women considering participation in the Sentinel Node Biopsy versus Axillary Clearance 2 breast cancer trial. Clin Trials 2015:12:409-17.

24. Fox J, Johns N, Lyons C, et al. PROforma: a general technology for clinical decision support systems. Comput Methods Programs Biomed 1997;54:59-67.

25. Sutton DR, Fox J. The syntax and semantics of the PROforma guideline modeling language. J Am Med Inform Assoc 2003;10:433-43.

26. Bury J, Hurt C, Roy A, et al. LISA: a web-based decision-support system for trial management of childhood acute lymphoblastic leukaemia. Br J Haematol 2005;129:746-54.

27. Taylor P, Fox J, Pokropek AT. The development and evaluation of CADMIUM: a prototype system to assist in the interpretation of mammograms. Med Image Anal 1999;3:321-37. 
28. Patkar V, Acosta D, Davidson T, et al. Using computerised decision support to improve compliance of cancer multidisciplinary meetings with evidence-based guidance. BMJ Open 2012;2: e000439.

29. Oken MM, Creech RH, Tormey DC, et al. Toxicity and response criteria of the Eastern Cooperative Oncology Group. Am J Clin Oncol 1982:5:649-55.

30. Collins IM, Kelleher F, Stuart C, et al. Clinical decision aids in colon cancer: a comparison of two predictive nomograms. Clin Colorectal Cancer 2012;11:138-42.

31. Trevena LJ, Zikmund-Fisher BJ, Edwards A, et al. Presenting quantitative information about decision outcomes: a risk communication primer for patient decision aid developers. BMC Med Inform Decis Mak 2013;13(Suppl 2):S7.

32. Elwyn G, O'Connor AM, Bennett C, et al. Assessing the quality of decision support technologies using the International Patient Decision Aid Standards instrument (IPDASi). PLOS ONE 2009;4: e4705.

33. Lewis CL, Golin CE, DeLeon C, et al. A targeted decision aid for the elderly to decide whether to undergo colorectal cancer screening: development and results of an uncontrolled trial. BMC Med Inform Decis Mak 2010;10:54.

34. Hersch J, Jansen J, Barratt A, et al. Overdetection in breast cancer screening: development and preliminary evaluation of a decision aid. BMJ Open 2014;4:e006016.

35. Bennett C, Graham ID, Kristjansson E, et al. Validation of a preparation for decision making scale. Patient Educ Couns 2010;78:130-3.
36. Miles A, Wardle J, Atkin W. Receiving a screen-detected diagnosis of cancer: the experience of participants in the UK flexible sigmoidoscopy trial. Psychooncology 2003;12:784-802.

37. Braun V, Clarke V. Using thematic analysis in psychology. Qual Res Psychol 2006;3:77-101.

38. Trevena LJ, Davey HM, Barratt A, et al. A systematic review on communicating with patients about evidence. J Eval Clin Pract 2006;12:13-23.

39. Zikmund-Fisher BJ, Fagerlin A, Ubel PA. Improving understanding of adjuvant therapy options by using simpler risk graphics. Cancer 2008;113:3382-90.

40. Zikmund-Fisher BJ, Angott AM, Ubel PA. The benefits of discussing adjuvant therapies one at a time instead of all at once. Breast Cancer Res Treat 2011;129:79-87.

41. Teufel A, Gerken M, Hartl J, et al. Benefit of adjuvant chemotherapy in patients with T4 UICC II colon cancer. BMC Cancer 2015;15:419.

42. Verhoeff SR, van Erning FN, Lemmens VE, et al. Adjuvant chemotherapy is not associated with improved survival for all high-risk factors in stage II colon cancer. Int J Cancer 2016;139:187-93.

43. Maak M, Simon I, Nitsche U, et al. Independent validation of a prognostic genomic signature (ColoPrint) for patients with stage II colon cancer. Ann Surg 2013;257:1053-8.

44. Gao S, Tibiche C, Zou J, et al. Identification and construction of combinatory cancer hallmark-based gene signature sets to predict recurrence and chemotherapy benefit in stage II colorectal cancer. JAMA Oncol 2016;2:37-45.

45. Fagerlin A, Pignone M, Abhyankar P, et al. Clarifying values: an updated review. BMC Med Inform Decis Mak 2013;13(Suppl 2):S8. 